\title{
Erratum to: Kenneth G. Wilson: Renormalized After-Dinner Anecdotes
}

\section{Paul Ginsparg}

Published online: 8 October 2014

(C) Springer Science+Business Media New York 2014

\section{Erratum to: J Stat Phys DOI 10.1007/s10955-014-1092-x}

As pointed out by M.E. Fisher, the last name of physicist Murray Gell-Mann was incorrectly transcribed in eleven of thirteen occurrences in the text. The correct spelling of the last name of Kenneth Wilson's thesis advisor is 'Gell-Mann', as above. I have great empathy for people whose last names are misspelled, and very much regret missing this error in the proofs.

The online version of the original article can be found under doi:10.1007/s10955-014-1092-x.

P. Ginsparg $(\bowtie)$

Cornell University, 452 Physical Sciences Building, Ithaca, NY14853, USA

e-mail: ginsparg@cornell.edu 